\title{
Pressure impulse theory for a slamming wave on a vertical circular cylinder
}

\author{
Ghadirian, Amin; Bredmose, Henrik
}

Published in:

Journal of Fluid Mechanics

Link to article, DOI:

10.1017/jfm.2019.151

Publication date:

2019

Document Version

Peer reviewed version

Link back to DTU Orbit

Citation (APA):

Ghadirian, A., \& Bredmose, H. (2019). Pressure impulse theory for a slamming wave on a vertical circular cylinder. Journal of Fluid Mechanics, 867, [R1]. https://doi.org/10.1017/jfm.2019.151

\section{General rights}

Copyright and moral rights for the publications made accessible in the public portal are retained by the authors and/or other copyright owners and it is a condition of accessing publications that users recognise and abide by the legal requirements associated with these rights.

- Users may download and print one copy of any publication from the public portal for the purpose of private study or research.

- You may not further distribute the material or use it for any profit-making activity or commercial gain

- You may freely distribute the URL identifying the publication in the public portal

If you believe that this document breaches copyright please contact us providing details, and we will remove access to the work immediately and investigate your claim 


\title{
Pressure impulse theory for a slamming wave on a vertical circular cylinder
}

\author{
Amin Ghadirian ${ }^{1} \dagger$, Henrik Bredmose ${ }^{1}$ \\ ${ }^{1}$ Department of Wind Energy, Technical University of Denmark, Nils Koppels Allé, Building \\ 403, DK-2800 Kgs. Lyngby, Denmark
}

(Received xx; revised xx; accepted $\mathrm{xx}$ )

A pressure impulse model is presented for wave impact on vertical circular cylinders. Pressure impulse is the time integral of the pressure during an impact of short time scale. The model is derived for a simplistic geometry and has the relative impact height, crest length and cylinder radius as effective variables. The last parameter, the maximum angle of impact is free and can be calibrated to yield the right force impulse.

A progression of simpler pressure impulse models are derived in terms of a 3D box generalization of the $2 \mathrm{D}$ wall model and an axisymmetric model for vertical cylinders. The dependence on the model parameters are investigated in the simpler models and are linked to the behaviour of the 3D cylinder model.

The model is next validated against numerical results for a wave impact for a phase- and direction-focused wave group. The maximum impact angle is determined by calibration against the force impulse. A good match of the pressure impulse fields is found. Further comparison to the force impulse of two common models in marine engineering reveals improved consistency for the present model. The model is found to provide a promising representation of the pressure impulse field, based on a limited number of input parameters. Its further validation and potential as a robust tool in force and response prediction is discussed.

Key words:

\section{Introduction}

In the design process of offshore structures the slamming wave load calculations play an important role. Several engineering models are commonly used in the industry as an extension to standard force models such as Morison (1953) and Rainey (1989, 1995, 2007). One of the most commonly used approaches for vertical circular cylinders is based on the work of Goda et al. (1966) formulated as

$$
F_{I}(t)=\lambda \eta_{b} \pi \rho R C^{2}\left(1-\frac{C}{R} t\right),
$$

where $\lambda \eta_{b}$ is the height of the impact zone, $\eta_{b}$ is the crest height of the breaking wave and $\lambda$ is defined as the curling factor. Further, $C$ is the wave celerity, $\rho$ is the fluid density and $R$ is the cylinder's radius. The time interval of this equation is from 0 to $R / C$. The breaker front is vertical and moves with the celerity of the wave. Very often the maximum inline force per unit height is described as a function of a slamming coefficient, $C_{s}$, where

$\dagger$ Email address for correspondence: amgh@dtu.dk 
$F_{I, \max }=C_{s} \rho R V^{2}$. In the formulation of Goda et al. (1966), $C_{s}=\pi$, according to the von Karman (1929) model, which does not account for deformation of the free surface. The latter causes the immersion of the cylinder to occur earlier, resulting in a decrease of the impact duration and an increase of the impact peak force/pressure. This behaviour is included in the model of Wagner (1932) who developed a solution for the peak pressure during the impact of a solid object on a still fluid in two dimensions. The solution predicted a slamming coefficient of $C_{s}=2 \pi$. Later Cointe \& Armand (1987) applied the method of matched asymptotic expansions to solve the boundary condition problem of the velocity potential and free surface of the flow around the structure. The model provides the same result as Wagners theory for the slamming coefficient, $C_{s}=2 \pi$. The study further included the decay of the impact force on the cylinder in time. More recently, Wienke \& Oumeraci (2005) proposed a new formulation which extends the model of Wagner (1932) for the total duration of the impact in three dimensions. Hallowell et al. (2016) used measurements from a site test campaign of the Blyth wind farm off the coast of England to categorize and identify breaking waves and quantify the variability of their impact loads. The accuracy of several breaking wave criteria and impact force models were analysed, and the impact force models were shown to represent the measurements with varying accuracy and to be sensitive to modelling assumptions. Their results showed that the mudline moments calculated using the model developed by Goda et al. (1966) were most consistent with the measurements, however, this consistency was claimed to be site and structure specific.

Since the duration of the impact on the structure is usually very short, the time integrated force impulse may be sufficient for an accurate response prediction. The pressure impulse theory is relevant in such cases. Further the theory gives the spatial distribution of the pressure impulse over the structure. In Cooker \& Peregrine (1995), the pressure impulse theory was derived for wave impact on a vertical wall in $2 \mathrm{D}$. A parametric study was performed to identify the dependency to the ratio of the breaker height to total depth and the length of the breaker. Later this model was extended to 3D cases by Wood \& Peregrine (1998). The model was not validated against measurements or other more comprehensive models. The approach was extended to more complicated configurations such as breaking wave impact on a wall by Iafrati \& Korobkin (2006), impact on an elastic wall by Korobkin \& Malenica (2007), wave impact on perforated structures by Korobkin (2008) and breaking wave impact on permeable barriers by Cooker (2013). In a more general form, Scolan \& Korobkin (2001) addressed the threedimensional problem of blunt-body impact onto the free surface of an ideal incompressible liquid within the Wagner theory. The method provides a wide range of exact analytical solutions in the case of an elliptic contact region. They suggested to use strip theory for elongated cases such as cylinders. The work did not include validations against experiments. Korobkin \& Scolan (2006) addressed the same problem with asymptotic analysis of the linearised Wagner problem for close to axisymmetric bodies such as cones and pyramids. Korobkin (2004) reviewed the models available for the prediction of the hydrodynamic pressure distribution and the force on a body entering liquid. Particular attention was given to analytical models which are based on the velocity potential given by the classical Wagner theory. He modified the Wagner solution by including the nonlinear terms in the Bernoulli equation and compared the results between the original and modified Wagner solution to the original and modified Logvinovich model (Logvinovich \& Yakimov 1973; Korobkin \& Malenica 2005). He validated these results against simulations and experiments and concluded that the modified Logvinovich model is well suited for practical calculations of impact loads acting on an arbitrary blunt body. Most recently Chatjigeorgiou et al. (2016b) presented a three dimensional steep wave impact onto a 
vertical plate with finite width and obtained the time variation of the slamming load on a vertical cylinder by combination with strip theory. Later they validated the model against lab data for a three-dimensional wave impact onto a rectangular column with the wave front resembling the face of a steep wave (Chatjigeorgiou et al. 2016a). In a further paper, Chatjigeorgiou et al. (2017) treated the three-dimensional hydrodynamic slamming problem on a vertical cylinder as a set of 2-D problems in the vertical direction. They found a closed form solution for the time dependent potential, the pressure impulse, the shape of the wave front and the slamming force as a function of time. More thorough review of the previous works can be found in the review papers presented by Peregrine (2003), Faltinsen et al. (2004) and Dias \& Ghidaglia (2018).

In the present paper a $3 \mathrm{D}$ pressure impulse model for a wave impact of a wedgeshaped geometry is derived. A 3D box generalization of the $2 \mathrm{D}$ wall model of Cooker \& Peregrine (1995) is presented $(\S 2)$ along with an axisymmetric impact model. The effective parameters of the $3 \mathrm{D}$ impact model are investigated with reference to the behaviour of the two simpler models. The role of the maximum impact angle, and of the model parameters, is discussed and a comparison to a realistic numerical wave impact is presented (§3). A summary and further discussion of the model applications are presented in $(\S 4)$.

\section{Mathematical formulation}

The peak pressure of an impulse event can vary substantially between impacts with the same nominal characteristics. Linked to the overall momentum conservation, however, the time integral of the pressure - the pressure impulse - can be expected to show a smaller variability. Hence, Cooker \& Peregrine (1995) suggested that pressure impulse is a better quantity to model an impact event than the peak pressure. Further, for impacts of small duration relative to the structures natural frequency, the response is governed by the impulse rather than the detailed time history of force and pressure. The pressure impulse $P$ is defined by

$$
P(\mathbf{x})=\int_{t_{b}}^{t_{a}} p(\mathbf{x}, t) d t
$$

where $t_{b}$ and $t_{a}$ are the times immediately before and after impact respectively and pressure is denoted by $p$. The speed of the fluid is often much less than the speed of sound so the incompressibility assumption can be applied. In addition, the effect of viscosity is negligible. Further, since the impact occurs in a very short time, the convective terms and gravity are negligible so Euler's equations read

$$
\frac{\partial \mathbf{u}}{\partial t}=-\frac{1}{\rho} \nabla p
$$

After integration with respect to time through the impact interval the equation becomes

$$
\underline{u}_{a}-\underline{u}_{b}=-\frac{1}{\rho} \nabla P .
$$

This equation can be rewritten by taking the divergence of both sides, which leads to the Laplace equation for the pressure impulse $\nabla^{2} P=0$. Following Cooker \& Peregrine (1995), the pressure impulse field satisfies the boundary conditions $P=0$ at a free surface, $\partial P / \partial n=0$ at a stationary rigid body boundary and $\partial P / \partial n=\rho u_{n b}$ where the liquid meets a solid boundary during impact. Here $u_{n b}$ is the fluid impact velocity projected to the normal direction of the boundary. 


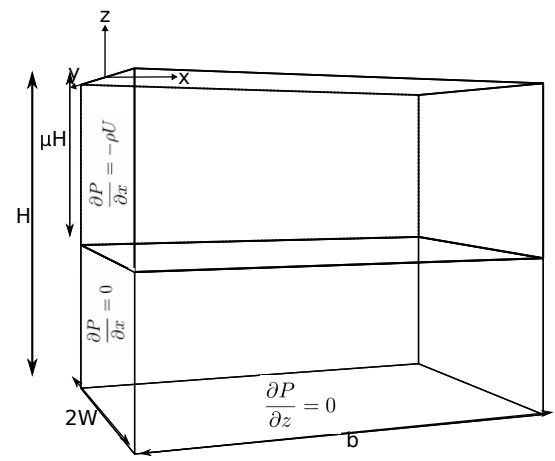

Figure 1: Definition sketch for 3D block impact on a flat plate.

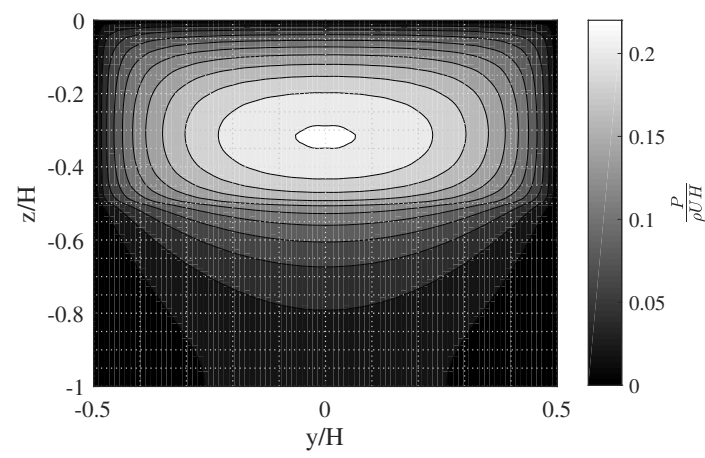

Figure 2: Pressure impulse of a finite-width fluid block on a flat vertical plate at $\mathrm{x}=0$ for $\mu=0.5, W / H=0.5$ and $b / H=1$.

\subsection{Impact of an idealized finite-width wave on a vertical wall}

The simplest case in three dimensions is to have a box shaped volume of fluid impacting a flat vertical wall. The domain and the boundaries for this problem is shown in figure 1 . The flat plate is located at $x=0$. At the time of impact, the fluid touches the wall in the region $-H \leqslant z \leqslant-\mu H$ and hits the wall with normal velocity $U$ in the region $-\mu H \leqslant z \leqslant 0$. Note that $\mu$ is defined as the ratio of the height of the impacting fluid to the whole fluid height until the bed. Hence, it is slightly different from the curling factor, $\lambda$, which is associated with the crest height. Other limits of the domain are $0 \leqslant x \leqslant b$ and $-W \leqslant y \leqslant W$. The boundary conditions on the flat plate and on the bed are shown in the figure. On the other boundaries $P=0$ must be satisfied.

The Laplace equation is solved using separation of variables and Fourier series analysis. The solution can be written as

$$
P(x, y, z)=\sum_{m=1}^{\infty} \sum_{n=1}^{\infty}\left(A_{m n} \cos \left(L_{m} \frac{y}{W}\right) \sin \left(k_{n} \frac{z}{H}\right) \frac{\sinh \left(\sqrt{L_{m}^{2}\left(\frac{H}{W}\right)^{2}+k_{n}^{2}}\left(\frac{b}{H}-\frac{x}{H}\right)\right)}{\cosh \left(\sqrt{L_{m}^{2}\left(\frac{H}{W}\right)^{2}+k_{n}^{2}} \frac{b}{H}\right)}\right),
$$

where $L_{m}=(m-1 / 2) \pi, k_{n}=(n-1 / 2) \pi$, and

$$
A_{m n}=4 \rho U H \frac{\left(\cos \left(k_{n} \mu\right)-1\right) \sin \left(L_{m}\right)}{k_{n} L_{m} \sqrt{L_{m}^{2}\left(\frac{H}{W}\right)^{2}+k_{n}^{2}}} .
$$

Similar results for the same problem, were presented by Wood \& Peregrine (1998). For $W \rightarrow \infty,(2.4)$ and (2.5) reduces to the 2D solution of Cooker \& Peregrine (1995). Additional to the 2D properties, (2.4) has an extra dependency to the second horizontal direction through the term $\cos \left(L_{m} y / W\right)$ and the coefficients in the hyperbolic functions. The normalized pressure impulse field can thus be seen to depend on three dimensionless parameters as follows:

$$
\frac{P}{\rho U H}\left(\frac{x}{H}, \frac{y}{W}, \frac{z}{H}\right)=f\left(\frac{b}{H}, \mu, \frac{W}{H}\right) .
$$

The parameters are the relative length of the impacting block $b / H$, the relative height of the impact zone $\mu$ and the relative width of the block $W / H$. As an example, the result for $\mu=0.5, W / H=0.5$ and $b / H=1$ is shown in figure 2 . The pressure impulse extends in the middle of the block to lower heights than $-\mu H$ which means that part of the 


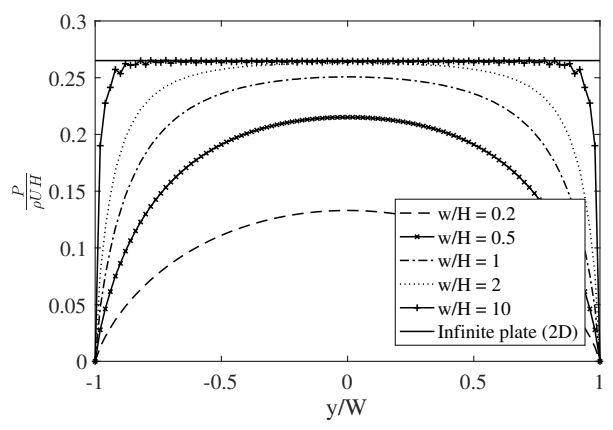

Figure 3: Pressure impulse as a function of width of the impacting block, plotted in the mid-height on the flat wall $(z / H=-\mu / 2$ and $x / H=0)$.

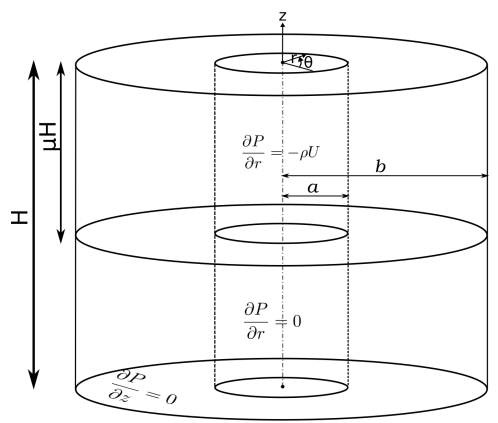

Figure 5: Definition sketch for axisymmetric impact on a vertical cylinder.

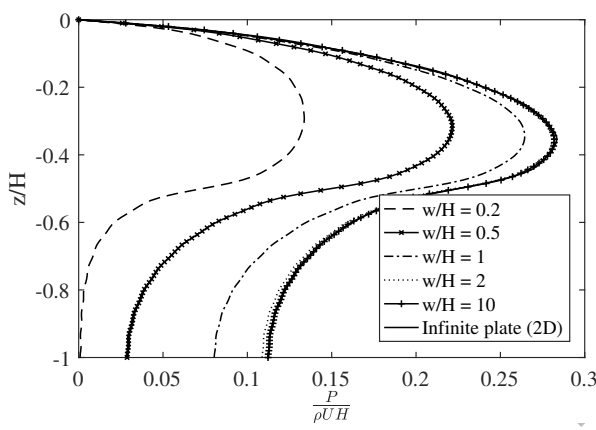

Figure 4: Pressure impulse as a function of width of the impacting block, plotted in the mid-width on the flat wall $(y / W=0$ and $x / H=0)$.

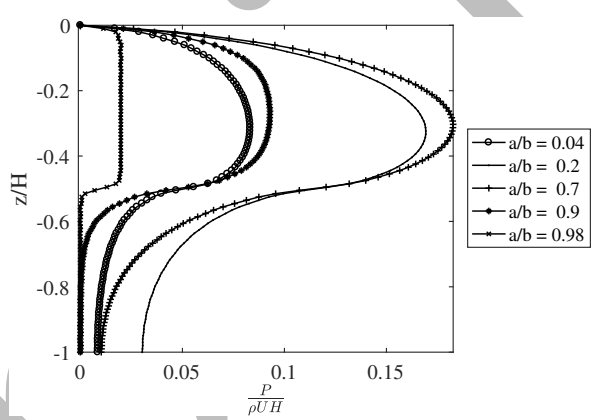

Figure 6: Pressure impulse of a axisymmetric wave impact on a cylinder with increasing $a / b$.

pressure impulse is carried by the bottom fluid. Convergence was achieved in this case with 30 components in each summation in (2.4).

The dependency of the pressure impulse on the width of the impacting block of fluid is shown in figure 3 in the depth of $z / H=-\mu H / 2$ as a function of $y / W$. The pressure impulse is maximum at the centre of the plate $(y / W=0)$ and increases with width until the $2 \mathrm{D}$ limit is reached as marked by a solid line. At the middle of the plate this is achieved for $W / H \approx 2$ in the present case and is gradually achieved for larger $W / H$ values towards the sides of the wall. In figure 4 the pressure impulse at the vertical centre line of the plate is plotted as a function of height for the same parameters. As the width of the fluid box is increased to $W / H=10$, the results of the box impact approaches the $2 \mathrm{D}$ case results.

\subsection{Impact of an idealized all-directional wave on a cylinder}

With the insight from the 3D box impact problem we proceed to impacts on cylinders. First the simple case of an axisymmetric impact is studied. An idealized wave front hits the cylinder from all directions with a normal velocity of $U$ in the vertical zone $-\mu H \leqslant z \leqslant 0$. Below this zone, in $-H \leqslant z \leqslant-\mu H$, the fluid touches the cylinder at impact. The outer radius of the impacting fluid is denoted by $b$, and the cylinder radius by $a$. A sketch of the domain and the boundary conditions is provided in figure 5 . The boundaries on the walls are shown in the figure while at all other boundaries $P=0$. 
(a)

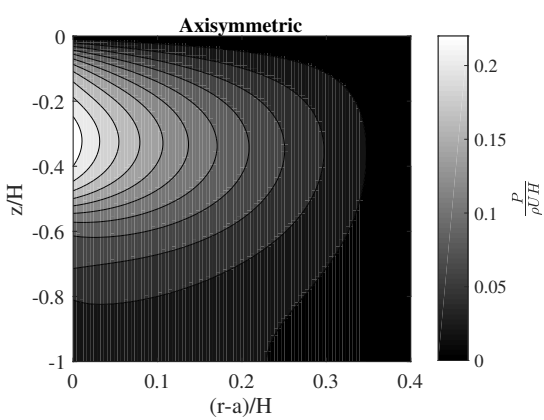

(b)

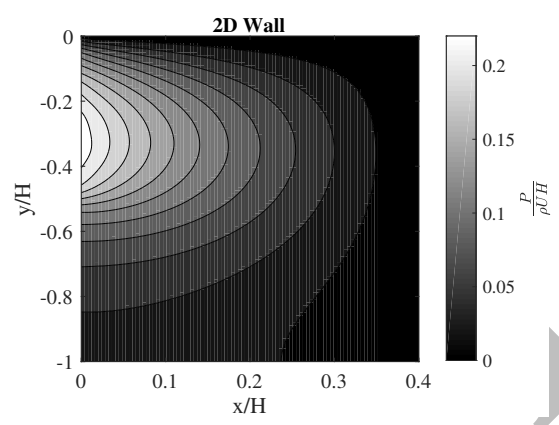

Figure 7: Pressure impulse (with $\mu=0.5$ ) for (a) an axisymmetric cylinder $(\mathrm{b} / \mathrm{H}=10.4$ and $\mathrm{a} / \mathrm{b}=0.96)$ and $(\mathrm{b})$ a $2 \mathrm{D}$ vertical wall $(\mathrm{b} / \mathrm{H}=0.4)$.

The Laplace equation is solved in the cylindrical coordinate system to yield

$$
P=\sum_{n=1}^{\infty}\left(A_{n} \frac{I_{0}\left(k_{n} \frac{r}{H}\right)+\alpha_{n} K_{0}\left(k_{n} \frac{r}{H}\right)}{\partial_{r}\left(I_{0}\left(k_{n} \frac{r}{H}\right)+\alpha_{n} K_{0}\left(k_{n} \frac{r}{H}\right)\right)_{r=a}} \sin \left(k_{n} \frac{z}{H}\right)\right),
$$

where $k_{n}=(n-1 / 2) \pi, \partial_{r}$ is the partial derivative with regards to $r, I_{0}$ and $K_{0}$ are the first and second kind modified Bessel functions of zeroth order and

$$
A_{n}=2 \rho U \frac{1-\cos \left(k_{n} \mu\right)}{k_{n}}
$$

The modified Bessel functions $I_{0}$ and $K_{0}$ were chosen for the radial dependency due to their non-oscillatory variation, which is suitable for the impact problem. A linear combination of the two functions is used here to assure compliance with the boundary condition $P=0$ at $r=b$ using the multiplier

$$
\alpha_{n}=\frac{-I_{0}\left(k_{n} b / H\right)}{K_{0}\left(k_{n} b / H\right)}
$$

From (2.7), the non-dimensional pressure impulse on the cylinder wall depends on the relative outer radius of the impacting fluid $b / H$, the relative height of the impact region $\mu$ and the ratio of the inner to outer radius $a / b$ :

$$
\frac{P}{\rho U H}\left(\frac{r}{H}, \frac{z}{H}\right)=f\left(\frac{b}{H}, \mu, \frac{a}{b}\right) .
$$

The axisymmetric pressure impulse solution is shown in figure 6 for $b / H=1, \mu=0.5$ and varying radius ratio. When the inner radius is increased, the cylinder wall absorbs a larger amount of incident momentum and the pressure impulse increases. For the present values of $\mu$ and $b / H$, it reaches a maximum at $a / b$ around 0.7 and decreases thereafter due to the reduced initial momentum for $a / b \rightarrow 1$.

The axisymmetric solution can be compared to the $2 \mathrm{D}$ wall case of Cooker \& Peregrine. Good similarity can be expected when the radius of the cylinder is so large that the effect of curvature, $1 / a$, is negligible. The pressure impulse field for the axisymmetric case of $b / H=10.4, a / b=0.96$ and the wall case of $b / H=0.4$ are compared in figure 7 . For both cases $\mu=0.5$. A very similar appearance of the two fields can be seen.

\subsection{Impact of an idealized wave on a cylinder with azimuth limits}

We now proceed to the 3D model of impact on a cylinder. The domain of the problem and its boundary conditions are shown in figure 8 . In this case, the fluid is wedgeshaped in the azimuthal direction with limits $-\theta_{\max } \leqslant \theta \leqslant \theta_{\max }$. In the lower region 


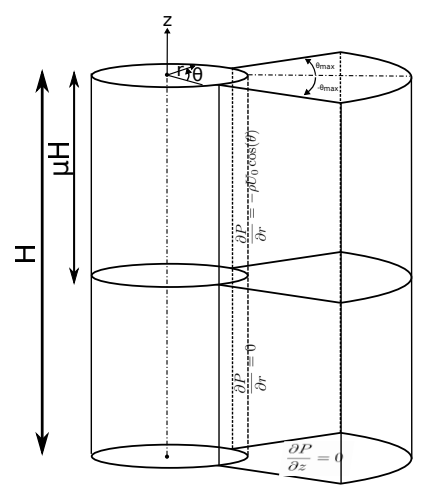

Figure 8: Definition sketch for wedge-shaped 3D impact on a vertical cylinder.

$-H \leqslant z \leqslant-\mu H$ the fluid touches the cylinder, while in the upper region $-\mu H \leqslant z \leqslant 0$ it impacts on the cylinder with velocity $U \cos (\theta)$ in the negative radial direction. At boundaries $\theta= \pm \theta_{\max }, z=0$ and $r=b$ the condition $P=0$ is satisfied. The Laplace equation is solved in the cylindrical coordinate system to yield

$$
P=\sum_{m=1}^{\infty} \sum_{n=1}^{\infty}\left(A_{m n} \cos \left(L_{m} \theta / \theta_{\max }\right) \sin \left(k_{n} \frac{z}{H}\right) \frac{I_{q_{m}}\left(k_{n} \frac{r}{H}\right)+\alpha_{m n} K_{q_{m}}\left(k_{n} \frac{r}{H}\right)}{\partial_{r}\left(I_{q_{m}}\left(k_{n} \frac{r}{H}\right)+\alpha_{m n} K_{q_{m}}\left(k_{n} \frac{r}{H}\right)\right)_{r=a}}\right)
$$

where $L_{m}=(m-1 / 2) \pi, k_{n}=(n-1 / 2) \pi$ and $q_{m}=L_{m} / \theta_{\max }$ is the order of the Bessel functions. Further $\alpha_{m n}$ is chosen such that $P=0$ at $r=b$,

$$
\alpha_{m n}=\frac{-I_{q_{m}}\left(k_{n} b / H\right)}{K_{q_{m}}\left(k_{n} b / H\right)}
$$

and

$$
A_{m n}=\frac{2 \rho U}{\theta_{\max }} \frac{1-\cos \left(k_{n} \mu\right)}{k_{n}} \int_{-\theta_{\max }}^{\theta_{\max }} \cos (\theta) \cos \left(L_{m} \theta / \theta_{\max }\right) d \theta d z .
$$

In this case the non-dimensional pressure impulse depends on the maximum impact angle, $\theta_{\max }$, in addition to the parameters already defined for the axisymmetric impact:

$$
\frac{P}{\rho U H}\left(\frac{r}{H}, \theta, \frac{z}{H}\right)=f\left(\frac{b}{H}, \mu, \frac{a}{b}, \theta_{\max }\right) .
$$

A parameter study was conducted to investigate the effect of the different parameters. The dependence on the relative length of the impacting wave $b / H$ is shown in figure 9 (a) for $\theta=0, \mu=0.5, a / H=0.1$ and $\theta_{\max }=\pi / 4$. As $b / H$ increases up to 0.35 the pressure impulse increases in all heights and then remains unchanged. This shows an asymptotic behaviour for increasing $b / H$. The same asymptotic behaviour was observed by Cooker \& Peregrine (1995) for the 2D flat plate case. We next investigate the dependence on the height of the impact region, $\mu$, in figure $9(\mathrm{~b})$. A width of $b / H=0.3$ is used and $\mu$, $\theta_{\max }$ and $a / H$ are identical to the values of figure $9(\mathrm{a})$. By the increase of $\mu$, the peak of the pressure impulse moves down as expected and increases the pressure impulse. The results from this figure are also consistent with the ones shown in figure 5 of Cooker \& Peregrine (1995).

The variation with respect to the relative inner radius $a / b$ is investigated in figure 10 (a) for $\mu=0.5, \theta_{\max }=\pi / 4$ and $b / H=0.3$. The pressure impulse increases as $a / b$ 
(a)

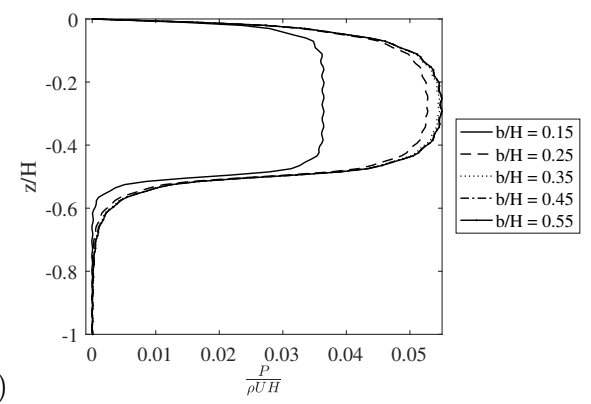

(b)

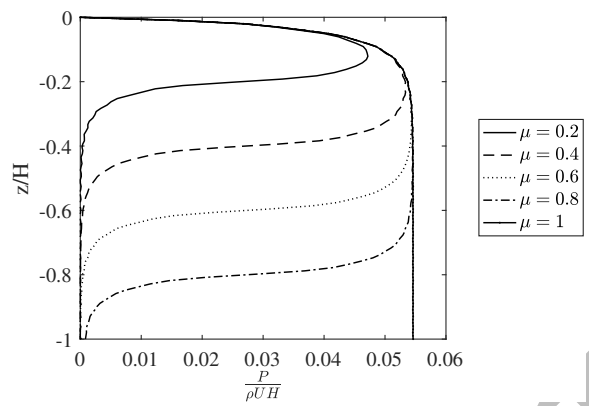

Figure 9: Dimensionless pressure impulse on the inner cylinder, at $\theta=0$, plotted as function of $z / H$ (a) for several values of $b / H$ and (b) for several values of $\mu$.

(a)
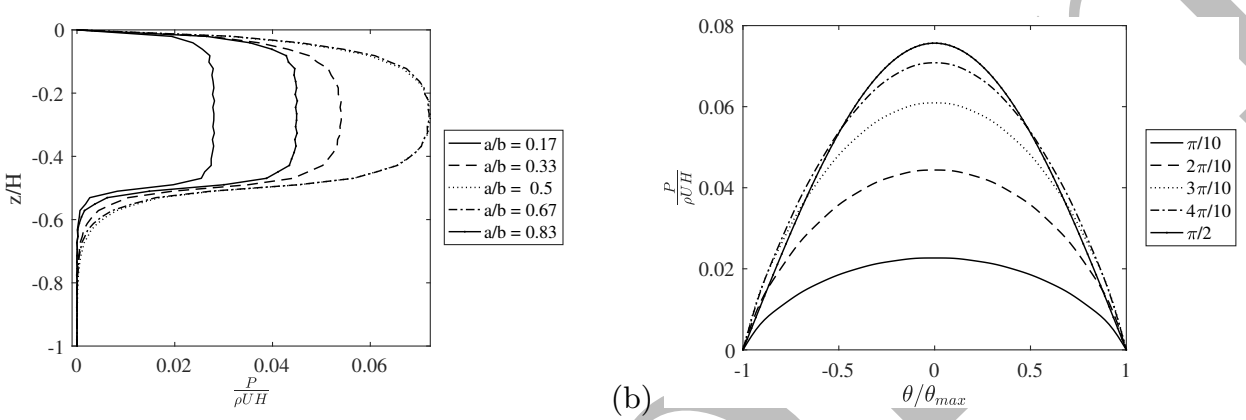

(b)

Figure 10: Dimensionless pressure impulse on the inner cylinder plotted (a) as function of $z / H$, at $\theta=0$, for several values of $a / b$ and (b) as function of $\theta / \theta_{\max }$, at $z=-0.25 \mu H$, for several values of $\theta_{\max }$.

increases up to $a / b=0.5$. For $a / b$ values larger than 0.67 the pressure impulse decreases similarly to the case shown for the axisymmetric impact. First, the area on the cylinder that absorbs incident momentum increases as the radius increases. Hereby the pressure impulse increases. However, at the same time the volume of fluid that impacts on the cylinder decreases so within the interval $0.5<a / b<0.7$, a value of $a / b$ occurs for which the distribution possesses a global maximum in pressure impulse.

The effect of the width of the fluid domain (in azimuth) is shown in figure 10(b) for $a / b=0.33, b / H=0.3$ and $\mu=0.5$. As the width of the domain increases the pressure impulse also increases. This increase can be explained by the increase in the total impacting volume of fluid $V=\left(b^{2}-a^{2}\right) \theta_{\max } \mu H$ as $\theta_{\max }$ increases. This conclusion is similar to the increased pressure impulse of the $3 \mathrm{D}$ impact of the fluid on the flat vertical plate of figure 3 . The rate of increase of the pressure impulse becomes gradually smaller as the azimuthal angle limit increases until it reaches a maximum at $\theta_{\max }=\pi / 2$.

\section{Validation against CFD results}

We now validate the model against a realistic wave impact. The chosen wave is a phaseand direction-focused wave group for a sea state of $H_{s}=9.5 \mathrm{~m}$ and $T_{p}=12 \mathrm{~s}$ in $33 \mathrm{~m}$ water depth with a cylinder diameter of $7 \mathrm{~m}$. CFD results at scale 1:50 for this impact were presented by Ghadirian et al. (2016). A snapshot of the wave at the time of impact is shown in figure 11. The impacting water spreads to the sides, upwards and downwards after impacting the monopile. Here $b$ is chosen as the distance from the cylinder centre to the back of the wave crest at the still water level. Further $\mu$ is determined from the 


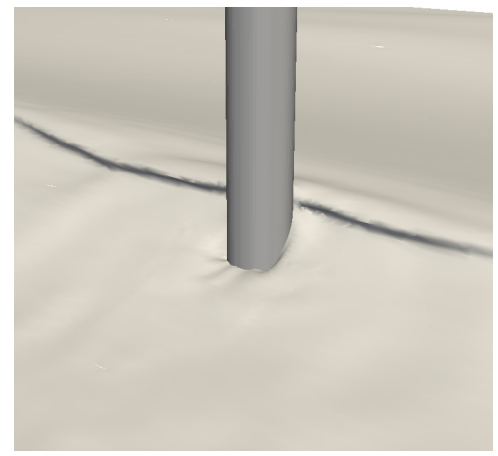

Figure 11: Snapshot of the wave impact on the cylinder from CFD model results.

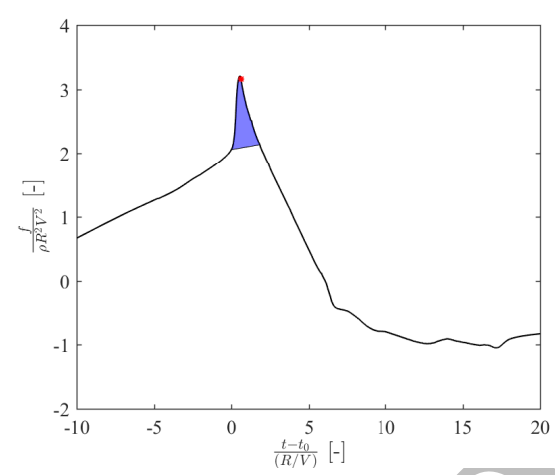

Figure 12: Inline force time series from the CFD model. The dashed blue region shows the effect of slamming.

height of the breaker. Hereby $b / H=0.64, \mu=0.12, a / b=0.13$ with $\theta_{\text {max }}$ left as the only free parameter.

In figure 12 the inline force time series from the CFD model is presented. The slamming contribution to the force time series is shown by blue shade and the time instant of the snapshot of figure 11 is marked by a red star. The area of the blue region is the same as the integrated pressure impulse on the cylinder wall after projection to the inline direction.

As a necessary step to isolate the impact force impulse from the contribution induced by the non-impacting parts of the wave, the pressure on the cylinder from just before the impact was subtracted from all the time instants in the CFD results. Then the time integral from the beginning of the impact until the end was calculated. The resulting pressure impulse is shown in figure 13(a). The largest pressure impulse is observed below $z / H=-0.05$ at $\theta / \theta_{\max }=0$ and the pressure impulse extends down to $z / H=-0.2$. Note that despite the subtraction of the pressures just before impact, some of the extended impulse may be caused by the kinematics of the wave itself and not necessarily the slamming impact. It is obseryed that the pressure impulse decreases to around zero at close to $\theta_{\max }$ which shows that the chosen azimuthal limit is sensible.

The result of the pressure impulse model can be seen in figure 13(b). The value of $\theta_{\max }$ was chosen such that the impact force impulse of the CFD model was equal to the wall-integrated pressure impulse in the inline direction. This led to $\theta_{\max }=\pi / 4$ with only $3 \%$ over-prediction of the impulsive force by the model. A general good consistency between the model and CFD pressure impulse distribution and magnitude is observed. The pressure impulse is localized in the upper layer in both the CFD and model results which is linked to the small breaker height ratio.

The model result is based on a fit of $\theta_{\max }$ to match the force impulse. However, since the force impulse is not generally available from the incident wave parameters, the dependency of the total impulsive force to $\theta_{\max }$ is shown in figure 14 . The total impulsive force increases as the azimuthal angle limit increases up until $\pi / 2$ where it reaches its maximum. The variation of the pressure impulse relative to $\theta_{\max }$ is robust and homogeneous. Hence, calibration based on this parameter is possible for similar cases. The total force impulse of the model can be compared to the existing impact models of Goda et al. (1966) and Wienke \& Oumeraci (2005). The force impulse calculated based on these models has about $190 \%$ and $100 \%$ over-prediction respectively. The over-prediction 
10
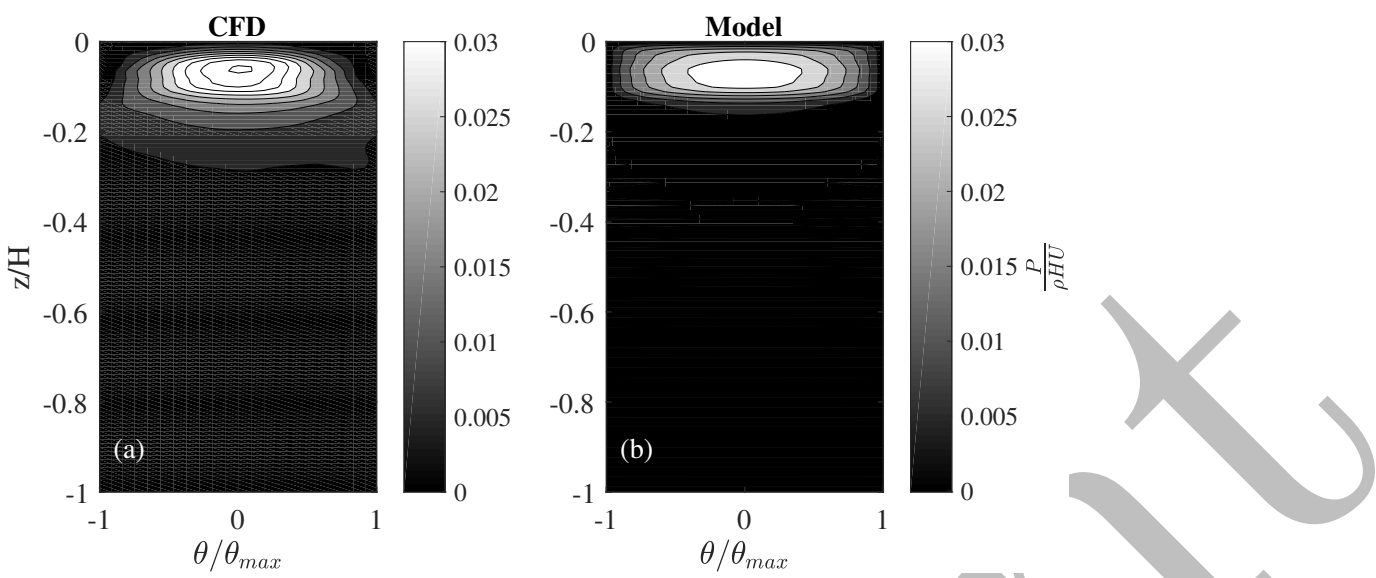

Figure 13: Pressure impulse contour plot from impact of a wave on a cylinder from (a) CFD model and (b) suggested pressure impulse model.

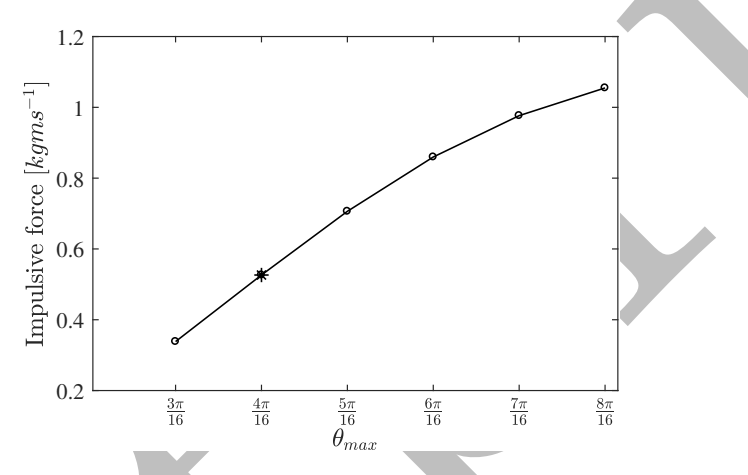

Figure 14: Total impulsive force as a function of $\theta_{\max }$. The star marker shows the value of force impulse for $\theta_{\max }=\pi / 4$ which was used for validation against CFD.

may be caused by the predicted maximum force magnitude as well as the time span, which has been validated to a lesser extent in previous studies.

\section{Summary and future work}

Pressure impulse models for a 3D box, axisymmetric impact on a vertical circular cylinder and three-dimensional wave impact on a vertical circular cylinder have been derived. The 3D model is formulated in a simplified wedge-shaped geometry and depends on the normalized crest length, impact zone height, inner radius and maximum impact angle. It is intended for calculation of slamming loads as an extension to standard force models such as Morison (1953) and Rainey $(1989,1995,2007)$. The parameter space was investigated and linked to the behaviour within the simpler models. We found that the pressure impulse increases with crest length up until an asymptotic limit, which appear to be effective for realistic wave impacts. The impact zone height governs the vertical extent of the pressure impulse distribution. The pressure impulse was further found to increase with increasing inner radius, $a$, up to a certain limit due to the increased cylinder wall area. However, after this limit the pressure impulse was found to decrease due to the diminishing incident momentum as the volume of the slamming water becomes smaller. The only parameter that can not directly be determined from the incident wave 
geometry is the maximum impact angle $\theta_{\max }$. In validation against a numerical wave impact, a value of $\theta_{\max }=\pi / 4$ was chosen through match of the numerical force impulse. This led to an encouraging agreement between the results of the two methods. For the same wave parameters, the force impulse was over-predicted by $190 \%$ and $100 \%$ by two established force models. This underlines the models potential for further validation and application in structural load and response calculations. The model shows robust and physics-inspired variations with respect to simple input parameters. Further work is thus to validate the model against more CFD results of impact events and experimental data. Ideally, repeated experiments of extreme waves with a structure, measuring the inline force, and without the structure, measuring the wave celerity, $\mu$ and $H$ are needed for such cases. Hereby more insight into the appropriate value of $\theta_{\max }$ can be gained and the probability distribution of test results relative to the models impulse prediction can be quantified.

\section{Acknowledgement}

This work was funded by the Innovation Fund Denmark and other partners as part of DeRisk project with grant number 4106-00038B. This support is gratefully acknowledged by the authors.

\section{REFERENCES}

Chatjigeorgiou, I. K. , Cooker, M. J. \& Korobkin, A. A. 2016a Three-dimensional water impact at normal incidence to a blunt structure. Proceedings of the Royal Society $A$.

Chatjigeorgiou, I. K. , Korobkin, A. A. \& Cooker, M. J. 2017 Three-dimensional steep wave impact on a vertical plate with an open rectangular section. International Journal of Mechanical Sciences 133 (4), 260-272.

Chatjigeorgiou, I. K. , Korobkin, A. A. , Cooker, M. J. \& Ave, P. $2016 b$ Threedimensional steep wave impact onto a vertical plate of finite width. In 31st IWWWFB, 3-6 April, 2016, Plymouth, Michigan, USA.

Cointe, R. \& Armand, J.-L. 1987 Hydrodynamic Impact Analysis of a Cylinder. Journal of Offshore Mechanics and Arctic Engineering 109 (3), 237-243.

Cooker, M. J. 2013 A theory for the impact of a wave breaking onto a permeable barrier with jet generation. Journal of Engineering Mathematics 79 (1), 1-12.

Cooker, M. J. \& Peregrine, H. 1995 Pressure-impulse theory for liquid impact problems. Journal of Fluid Mechanics 297, 193-214.

Dias, F. \& Ghidaglia, J. 2018 Slamming: Recent Progress in the Evaluation of Impact Pressures. Annual Review of Fluid Mechanics 50 (September 2017), 243-273.

Faltinsen, O. M. , Landrini, M. \& Greco, M. 2004 Slamming in marine applications. Journal of Engineering Mathematics 48, 187-217.

Ghadirian, A. , Bredmose, H. \& Dixen, M. 2016 Breaking phase focused wave group loads on offshore wind turbine monopiles. Journal of Physics: Conference Series $\mathbf{7 5 3}$ (9), 092004.

Goda, Y. Haranaka, S. \& Kitahata, M. 1966 Study on impulsive breaking wave forces on piles. Tech. Rep..

Hallowell, S. , Myers, A. T. \& Arwade, S. R. 2016 Variability of breaking wave characteristics and impact loads on offshore wind turbines supported bymonopiles. Wind Energy 19, 301-312.

Iafrati, A. \& Korobkin, A. 2006 Breaking wave impact onto vertical wall. In Proc. 4th Int. Conf. Hydroelas. Mar. Tech., Wuxi, China, 10-14 September, pp. 139-148.

von Karman, T. 1929 The impact on seaplane floats during landing. Tech. Rep.. National advisory committee for aeronautics. 
Korobkin, A. 2004 Analytical models of water impact. European Journal of Applied Mathematics 15 (6), 821-838.

Korobkin, A. 2008 Non-Classical Boundary Conditions in Water-Impact Problems. In IUTAM Symposium on Fluid-Structure Interaction in Ocean Engineering, pp. 167-178. Dordrecht: Springer Netherlands.

Korobkin, A. A. \& Malenica, S. 2005 Modified logvinovich model for hydrodynamic loads on asymmetric contours entering water. In International Workshop on Water Waves and Floating Bodies 2005.

Korobkin, A. A. \& MalenicA, S. 2007 Steep wave impact onto elastic wall. In International Workshop on Water Waves and Floating Bodies, pp. 2-5.

Korobkin, A. A. \& Scolan, Y. M. 2006 Three-dimensional theory of water impact. Part 2. Linearized Wagner problem. Journal of Fluid Mechanics 549, 343-373.

Logvinovich, G. V. \& Yakimov, Y. L. 1973 Submergence of bodies in liquid with large velocities. In Proc. IUTAM Symp. on Non-Steady Flow of Water at High Speeds (ed. LI Sedov \& G. Yu. Stepanov), pp. 85-92.

Morison, J. R. 1953 The Force Distribution Exerted by Surface Waves on Piles. Tech. Rep..

Peregrine, D. H. 2003 WATER-WAVE IMPACT ON WALLS. Annual Review of Fluid Mechanics 35 (1), 23-43.

RAINEY, R. C. 1989 A new equation for calculating wave loads on offshore structures. Journal of Fluid Mechanics 204 (WW3), 295-324.

RAINEY, R. C. T. 1995 Slender-body expressions for the wave load on offshore structures. Proceedings of the Royal Society of London 450 (1939), 391-416.

RAiney, R. C. T. 2007 Weak or strong nonlinearity: The vital issue. Journal of Engineering Mathematics 58 (1-4), 229-249.

Scolan, Y. M. \& Korobkin, A. A. 2001 Three-dimensional theory of water impact. Part 1. Inverse Wagner problem. Journal of Fluid Mechanics 440, 293-326.

WAGNER, H. 1932 Über Stoß und Gleitvorgänge an der Oberfläche von Flüssigkeiten. Zeitschrift für Angewandte Mathematik und Mechanik 12 (4), 193-215.

Wienke, J. \& Oumeraci, H. 2005 Breaking wave impact force on a vertical and inclined slender pile - Theoretical and large-scale model investigations. Coastal Engineering $\mathbf{5 2}$ (5), 435-462.

Wood, D. J. \& Peregrine, D. H. 1998 Two and three-dimensional pressure-impulse models of wave impact on structures. Coastal Engineering pp. 1502-1515.

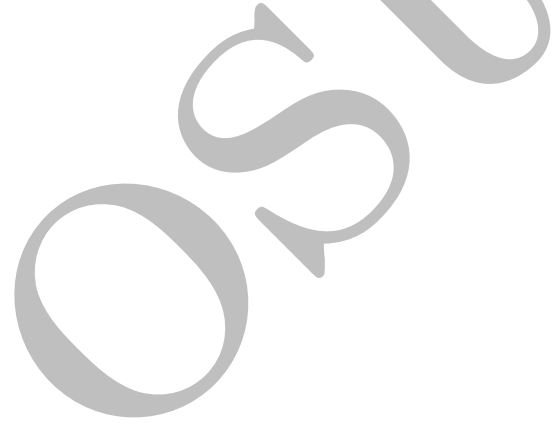

